\title{
UN CAMBIO DEMOCRÁTICO EN LAS AULAS UNIVERSITARIAS: UNA EXPERIENCIA EN LA FORMACIÓN DEL PROFESORADO DE EDUCACIÓN FÍSICA
}

\author{
Antonio Fraile Aranda \\ Universidad de Valladolid
}

RESUMEN: En primer lugar, este artículo muestra las diferentes definiciones relativas a cómo desarrollar una enseñanza democrática en el ámbito educativo. Para ello, presentamos unos principios pedagógicos sobre cómo atender la educación democrática, desde una perspectiva crítica, en las aulas universitarias.

A continuación explicamos cómo es una enseñanza democrática en el escenario universitario, referida a cuestiones morales, de control y poder del profesorado sobre los estudiantes, la atención a la diversidad, los conocimientos del profesor y la negociación entre profesor y estudiantes.

Para concluir, presentaremos una experiencia práctica de formación universitaria del profesorado de Educación Física, a partir de un proceso de democratización basado en acuerdos de negociación, metodologías activas desde un trabajo colaborativo y la autoevaluación a partir de los compromisos de los estudiantes.

ABSTRACT: The aim of this article is, first of all, to show the different definitions concerning the development of a democratic teaching in the classroom environment. For this reason, we put forward a series of pedagogical principles about how to reach a democratic education, from a critical perspective, at university level.

Secondly, we'll explain how a democratic teaching is being developed in universities, making explicit references to moral questions, teacher control over the students, bearing in mind the diversity, the knowledge of the teacher and the negotiation between him and his students.

Finally, we will present a practical experience of a university training for physical education teachers, beginning with a democratic process based in agreements of negotiation, active methodologies from a cooperative work and the self-assessment coming from the students compromises.

PALABRAS CLAVE: Democratización, enseñanza superior, formación de profesores, educación física 
KEYWORDS: Democratic education, higher education, proffesor training, phisical education

\section{INTRODUCCIÓN}

Para Marina y Válgoma (2000), la democracia representa una forma de gobernar los asuntos humanos a partir de considerar las opiniones de todos. A lo largo de la historia ésta ha ido evolucionando conectada a diferentes modelos sociales y a la experiencia comunicativa entre las personas.

A partir de una forma de gobernar asentada en la racionalidad, las relaciones entre educación y democracia se han incrementado, posibilitando mejores individuos sociales. Por tanto, la educación será el instrumento ideal que permita desarrollar las capacidades de pensar y participar en la construcción social, a través de un proceso democrático.

Son muchos los aspectos que inciden en un cambio del conocimiento y que condicionados por la globalización determinan una interdependencia económica, la extensión mundial de las comunicaciones y el crecimiento económico del mundo desarrollado. Dichos aspectos deben ser tratados y analizados desde la Universidad, configurando un plan de acción en el que se favorezca la interconexión de las redes locales, regionales y nacionales, estimulando el desarrollo y difusión de contenidos pedagógicos de interés europeo, promoviendo la formación de profesores, informando sobre las posibilidades pedagógicas de los medios audiovisuales y multimedia, estimulando la constitución de compromisos entre sectores públicos y privados, y movilizando las voluntades de los ciudadanos (Michavila y Calvo, 1998).

En el informe de la OCDE, del año 1996, sobre la educación se dice: que la rápida mutación de la economía y la técnica hace inútil una formación orientada exclusivamente hacia la formación profesional, pues la evolución de los conocimientos y las técnicas, y la propia transformación de las empresas deja rápidamente obsoleto su contenido. Además, la diversidad pluricultural de las sociedades postindustriales no acepta que se consideren a los centros escolares como entidades homogéneas; ante lo cual, los sistemas educativos deben ayudar al alumnado a adquirir una serie de saberes y competencias generales básicas, e inculcarles la capacidad de adaptarse al cambio, y sobre todo la actitud y el gusto por aprender durante toda su vida.

La educación debe ayudar a superar las tensiones entre lo mundial y lo local, lo universal y lo singular, el largo y el corto plazo, la indispensable competencia y la preocupación por la igualdad de oportunidades, el extraordinario desarrollo de los conocimientos y las capacidades de asimilación del ser humano, lo espiritual y lo material (Delors, 1996).

El incremento de los sistemas tecnológicos, la apertura a corrientes postmodernas, las pautas sociales que impulsan nuevos hábitos y costumbres entre los ciudadanos, llega a repercutir de forma directa en el ámbito de la educación. Esto determina que los docentes, a través tanto de la formación inicial como de la permanente, nos hagamos con nuevos modelos de intervención en el aula.

Más concretamente, entre las funciones de la enseñanza deben estar las de transmitir un volumen, cada vez mayor, de conocimientos teóricos y técnicos, adaptados 
a la sociedad y como base de las competencias del futuro. Asimismo, la enseñanza debe proporcionar las cartas náuticas de un mundo complejo y en perpetua agitación y, al mismo tiempo, la brújula para moverse por ese medio.

Para ello, y desde la Universidad, debemos preocuparnos no sólo por el cambio de contenidos, sino de los principios de procedimiento que posibiliten una actuación docente más democrática que resulte adecuada a las exigencias de esos cambios.

\section{2. ¿QUÉ ENTENDEMOS POR UNA EDUCACIÓN DEMOCRÁTICA?}

Una educación es democrática, para Dewey (1995), cuando se desarrolla entre los estudiantes un interés personal y crítico por lo social. Así, gracias a poder unir educación y democracia haremos estudiantes más autónomos y capaces de intervenir en la construcción social. Se trata de incorporar a las tareas formativas sus experiencias de la vida diaria y favorecer la participación colaborativa entre profesor y alumnos. Desde un curriculum democrático, en donde la participación de todos los agentes del centro sea libre, como un derecho básico que permita la organización social de la escuela. Esta visión, que se puede considerar ligada a una perspectiva crítica, ayuda a que los estudiantes se preocupen por los problemas sociales dentro de su proceso de aprendizaje.

"La enseñanza desde una perspectiva crítica facilita la transformación permanente del pensamiento, las actitudes y los comportamientos de los alumnos, provocando el contraste de sus adquisiciones más o menos espontáneas en su vida cotidiana con las proposiciones de las distintas disciplinas científicas, artísticas y especulativas, y también estimulando su experimentación en la realidad." (Pérez Gómez, 1992, pág. 81).

Para Marchesi (2000), el desarrollo del proceso democrático de los centros educativos exige de una participación general de todos los agentes sociales del entorno escolar, incluido el alumnado. Siendo preciso, implicar a todos ellos en el gobierno y en la toma de decisiones sobre cuestiones educativas que, hasta ahora, sólo eran potestad de los educadores. Por tanto, si apostamos por una escuela democrática, debemos promover el diálogo y las relaciones del grupo, corrigiendo las desigualdades sociales a partir de un proceso de deliberación que respete los principios éticos que faciliten la vivencia democrática de todos.

Según Martínez Rodríguez (1999), democratizar la escuela exige mejorar las condiciones para interpretar y aplicar estrategias que extiendan los derechos humanos entre los escolares. Por ello, en los centros de formación del profesorado hemos de preparar a los estudiantes hacia una escuela y un tipo de alumnado que debe aprender a pensar por sí mismos, dejando poco a poco de depender de una autoridad externa. Fomentando estrategias docentes que les ayude a que piensen por ellos mismos.

Todas estos cambios docentes deben partir de un principio de respeto y trato digno hacia los estudiantes, donde profesores y profesoras debemos: favorecer su toma de decisiones, asegurar la igualdad y la resolución de conflictos de forma democrática, establecer pautas de consulta, participación y decisión consensuada, utilizar el debate y la deliberación desde las diferentes posturas de los participantes, etc. No obstante, en ocasiones en la práctica, cuando estas propuestas entran en conflicto con nuestros principios y creencias, dificulta que los docentes acertemos 
sobre cómo orientar nuestro proceso hacia un tipo de enseñanza realmente democratizadora.

\section{3. ¿QUÉ TIPO DE DEMOCRACIA SE DESARROLLA EN EL ÁMBITO UNIVERSITA- RIO?}

Las vivencias en nuestro entorno académico nos dice que, en el escenario específico de la formación del profesorado, hay importantes carencias respecto a un tratamiento democrático en nuestras relaciones con los estudiantes; así, como en las propias relaciones entre el propio profesorado (debido a la situación de balcanización en que se encuentran los departamentos universitarios hoy día).

No es habitual que desde nuestros programas y desde nuestra acción didáctica, no sólo no ayudemos a los estudiantes a reflexionar sobre su práctica, sino que nuestra enseñanza se convierte en una transmisión, sin más, de unos contenidos en donde apenas tienen presencia las situaciones cambiantes del medio socioeducativo. Por ejemplo, desde nuestro modelo de enseñanza academicista no se enseña a los estudiantes a cuestionar o analizar las interconexiones entre cultura, poder y transformación, que les ayude a adquirir un mayor compromiso social ante las problemáticas vigentes en la sociedad.

Estas carencias se manifiestan, de forma especial, en los centros de formación del profesorado cuando el contenido que se transmite no considera, de una forma crítica, el estudio del contexto sociopolítico, cultural, económico, etc., de nuestra sociedad. Asimismo, desde esta formación no se ayuda suficientemente a los estudiantes a conectar la teoría y la práctica, a generar un pensamiento social preocupado por los valores, así como dotar de instrumentos a los estudiantes para que puedan generar un tipo de práctica más innovadora para la resolución de conflictos en el aula.

Martínez y Aróstegui (2001), señalan que en el espacio de enseñanza-aprendizaje no es habitual que los profesores y alumnos decidan de forma conjunta y democrática sobre los contenidos a aprender, sobre la metodología a utilizar en clase y sobre los criterios para la evaluación. Sin embargo sí es habitual para esos estudiantes (especialmente en el escenario universitario) que los profesores les impongan, de forma particular, el proceso educativo. Es decir, que les dicten los conocimientos, les marquen los ritmos y estilos para aprender, y la forma de evaluar y calificar sus aprendizajes.

Si nuestra tarea de enseñantes es favorecer una cultura democrática invitaremos al alumnado a que participen en el proceso de enseñanza y aprendizaje, incorporando sus perspectivas e intereses. Diseñando un curriculum que, además de contemplar sus preocupaciones, revise sus valores sociales. Asimismo, los docentes debemos reconocer nuestras carencias y errores, valorando los saberes, experiencias y vivencias del alumnado; siendo tan importante lo que se enseña, como la forma de hacerlo y para qué hacerlo.

Esta acción democratizadora en el aula se basará en facilitar que los estudiantes recuperen un papel más activo en su aprendizaje, diseñando estrategias de colaboración y rechazando las prácticas reproductoras de un conocimiento dogmático. Hacer que las decisiones sobre el programa surjan desde el debate y del diálogo permanente, entre docentes y estudiantes, como tarea de creación y construcción colaborativa (Contreras Domingo, 1995). 
Solo a través de una mejor conexión entre profesores y estudiantes, como parte de una comunidad de enseñanza-aprendizaje, se podrán conocer y compartir las necesidades e intereses de los aprendices, así como los principios y compromisos de los enseñantes. Escuchar y conversar con los estudiantes, como forma de intercambio, considerando sus preguntas y sus propuestas permitirá leer, según dice Hernández Alvarez (2000), la realidad social de la que todos, alumnos y docentes, formamos parte. Solo cuando los centros de formación del profesorado atiendan a una verdadera educación axiológica, podremos recuperar su función educativa y no sólo reproducir aquellos valores y actitudes socialmente vigentes (Bolívar, 1995).

Esto a su vez exige que los docentes asumamos una labor de investigadores de nuestra práctica, no sólo describiendo e interpretando las interacciones entre sociedad y educación, sino tratando de cambiarlas. Desde una teoría crítica, la función del docente no se entiende legitimando y justificando los hechos dominantes, sino interviniendo desde la reflexión y comprensión de la práctica, estudiando e identificando los valores socioeducativos. Donde profesores y estudiantes tengamos una mejor relación interpersonal, basada en la negociación, que nos lleve a establecer un programa que recoja sus necesidades e intereses.

Con ayuda de la Investigación-acción, los profesores debemos revisar lo que surge en la práctica, contrastando las observaciones e interpretaciones personales de la práctica escolar; así, como los factores que determinan los procesos que se desarrollan en el aula (Fraile, 1995). La finalidad de esta metodología, de la Investigación-acción, no es describir sino transformar nuestra realidad más inmediata elaborando alternativas para concebir, actuar y legitimar la práctica. Especialmente cuando estamos ante un tipo de Universidad donde algunos profesores optan por su supervivencia y seguridad, desde una enseñanza técnica hacia la eficacia.

Para Fernández Balboa (2000), esa actuación técnica se relaciona con un escaso compromiso ante los problemas sociales. El deseo de clarificar estas cuestiones, nos lleva a hacernos la siguiente pregunta: ¿Cómo podemos intervenir desde la Universidad para favorecer una escuela democrática y atender los nuevos problemas sociales?

\section{PRINCIPIOS EDUCATIVOS PARA DEMOCRATIZAR EL AULA UNIVERSITARIA}

Entre los principios educativos que deben impulsar un cambio democratizador en el aula universitaria deben estar, entre otros: el desarrollo del pensamiento crítico, la capacidad para colaborar con los otros y trabajar en equipo, el respeto hacia las opiniones ajenas, el sentido de la responsabilidad y del compromiso social (Fernández Balboa, 2000)

Una de las estrategias docentes, para facilitar una participación más democrática en las aulas, es ayudar a que los estudiantes adopten un papel más activo y crítico ante su aprendizaje, a través de acciones de colaboración que les permita trabajar en grupo y aprender unos de otros. Igualmente, desarrollar la negociación en las que se acuerde de forma conjunta: la finalidad de la enseñanza, el contenido a enseñar, la metodología y, de forma especial, la evaluación. Para ello, se debe procurar que las decisiones sobre cada uno de los componentes del programa surjan desde el debate y del diálogo permanente entre profesor y estudiantes, como tarea de creación y construcción colaborativa. En donde esté presente el respeto hacia las opiniones de los demás. 
Asimismo, desde esta perspectiva, el estudiante se aproximará a un mejor conocimiento de los contenidos del programa partiendo de su experiencia, intereses y conocimientos previos; no sólo modificando los conocimientos que posee, sino interpretando lo nuevo, integrándolo y haciéndolo suyo. Este proceso activo, parte de construir, modificar, enriquecer y diversificar sus esquemas de conocimiento. Por tanto, esta actividad de enseñanza-aprendizaje, ligada al modelo democrático, debe considerar los principios constructivistas de la teoría de la comunicación y del aprendizaje dialógico. Partiendo de los conocimientos previos de los estudiantes y de la deliberación como intercambio cognitivo, favoreciendo la comprensión, la reflexión crítica y el intercambio de sus conocimientos y experiencias (Vygostsky,1979; Coll, 1990).

Para Pozo (1996), el conocimiento debe representar una interacción entre la nueva información que se nos presenta y lo que ya sabíamos, y el aprender construir modelos que nos ayuden a interpretar la información que recibimos. Para ello, la acción educativa debe ajustarse a la situación y a las características de la actividad mental constructiva del alumnado, teniendo en cuenta que es preciso:

- Provocar desafíos que hagan cuestionar unos significados, acercando la comprensión y actuación del alumnado a las intenciones educativas.

- Plantear retos abordables para el alumnado, acompañados de apoyos y soportes intelectuales y emocionales, que les permita superar esas exigencias.

- Aumentar la capacidad de comprensión y actuación autónoma del alumnado.

Esa acción comunicativa, que se establece entre docente y estudiantes, deberá ser interactiva a partir de un planteamiento dialógico y colaborativo. Para ello, no se utilizará el diálogo como un medio para imponer la visión del docente a los demás, sino como forma de comunicación y de llegar a acuerdos. El diálogo como instrumento pedagógico debe facilitar la intersubjetividad y comunicación entre personas, favoreciendo una mejor relación entre teoría y práctica. Considerar que no hay verdades o soluciones definitivas sino que se deben ir construyendo respuestas provisionales, por medio de una dinámica intersubjetiva (Flecha, 1998).

Del mismo modo, para Pascual (1999), la formación del profesorado debe basarse en una aproximación del conocimiento a sus vidas, a su mundo, lo que ayudará a construir significados. Donde los docentes debemos considerar el principio de la igualdad de oportunidades, a través de escuchar, analizar e interpretar la participación de los estudiantes, considerando sus preguntas y propuestas, desde un lenguaje y una forma de actuar que respete su diversidad.

Asimismo, debemos promover la participación de los estudiantes, dándoles voz en diferentes momentos de la actividad docente. Considerando que la enseñanza democrática debe incorporar, como uno de sus contenidos básicos, la educación en valores, sin dejar de explicitar los criterios éticos que guían la actuación docente, donde los contenidos son más un medio que una finalidad docente. Mantener un tratamiento digno y respetuoso hacia los estudiantes, será para ellos un referente en su posterior actividad educativa. Según Rogers (1972), cuanto mayor sea la relación entre el profesor y el alumnado, menor importancia se atribuirá a los planteamientos dogmáticos y teóricos.

Por todo ello, la formación del profesorado debe atender el pensamiento democrático y la reflexión crítica de los estudiantes, ayudándoles a revisar sus experien- 
cias y vivencias previas, su dimensión afectiva, sus relaciones sociales, el desarrollo de la propia personalidad hacia la formación de una cultura propia.

\section{LA FORMACIÓN DEMOCRÁTICA EN EL ESCENARIO UNIVERSITARIO}

El proceso docente que venimos desarrollando un grupo de profesores, coincidentes con los anteriores principios y preocupados por una dimensión humanista, tiene como finalidad favorecer al alumnado una actuación más democrática en clase, sin olvidar que éstos adquieran un conocimiento teórico-práctico vinculado a su futura acción docente. El dominio hegemónico de un tipo de enseñanza academicista ha determinado que los profesores no hayan venido asumiendo, como habituales, una serie de conductas docentes favorecedoras de una mayor relación democrática. Por tanto, para cubrir esas carencias proponemos que los docentes deben favorecer:

a) Una mayor preocupación por los problemas morales relacionados con los derechos de los estudiantes. Los principios educativos de los profesores, como favorecedores de una intervención educativa democrática, deben estimular la libre circulación de ideas, la iniciativa individual y colectiva para resolver problemas, la reflexión crítica, el respeto a la dignidad y los derechos de las minorías. Para cumplir con estos principios, en vez de hacer declaraciones formales a favor de la igualdad de oportunidades, el respeto, la equidad, etc. deberíamos intervenir recogiendo en nuestras tareas docentes esos valores, desde una práctica que mejore esa relación democrática con los estudiantes y atendiendo su desarrollo moral. Por ejemplo, hay comportamientos docentes que conducen a la falta de respeto hacia los estudiantes, o que sólo atienden a las necesidades de aquellos a los que se les considera más capacitados o que coinciden con las expectativas del profesor.

b) Una mayor interacción con el alumnado superando el miedo a perder el control de la clase y las relaciones de poder. A los docentes se nos ha venido formando para evitar situaciones de incertidumbre, a través de diseñar programas y actividades homogéneas válidas para todos. Asimismo, se nos ha creado una preocupación especial por mantener el orden, la disciplina y por desarrollar la capacidad de imitar el conocimiento del experto. Incluso, de forma oculta, hemos diseñado actividades donde los objetivos, los contenidos, la metodología y la evaluación se han llevado a la práctica imponiendo nuestros criterios personales, como una muestra de poder y de no considerar las necesidades e intereses de los estudiantes.

Solo será posible negociar nuestra intervención educativa, cuando los profesores y alumnos compartamos entre iguales el poder. Poder que Devis (2001) interpreta como: la apropiación por parte del profesorado de la toma de decisiones relativas a la organización y al desarrollo del trabajo, que se lleva a cabo en las instituciones educativas. Así, desde la negociación democrática se posibilitará que tanto el profesor como los estudiantes estén en condiciones de igualdad para compartir sus compromisos.

Las propuestas para democratizar nuestra sociedad, transferibles al escenario educativo, pasan por mejorar las condiciones de igualdad entre los agentes educativos. Así, proponemos unos principios de procedimiento que ayuden en el desarrollo democrático de las aulas universitarias: 
- Establecer una relación respetuosa del profesorado con el alumnado.

- Ampliar y mejorar los métodos que favorezcan la toma de decisiones y la participación del alumnado.

- Favorecer la resolución democrática de conflictos como medio educativo.

- Diseñar métodos sistemáticos de consulta, participación y decisión colegiada.

- No defender autoritariamente posiciones personales.

- Utilizar el debate y la deliberación para abordar los temas controvertidos (negociando desde las diferentes posturas de los participantes).

- Asegurar la calidad y el nivel de conocimientos.

c) Una mayor atención a la diversidad de los estudiantes. Desde la formación del profesorado no se atiende suficientemente las estrategias docentes que ayuden a los docentes a cómo desarrollar la función compensatoria de las desigualdades de origen, desde la atención y respeto a la diversidad. Aunque la Universidad no puede anular esas discriminaciones, sí puede paliar sus efectos compensando las consecuencias individuales de la desigualdad social. Para ello, se debe diseñar un curriculum que permita la igualdad de oportunidades, desde una pedagogía di versificada en el marco de la enseñanza comprensiva que fomente la pluralidad de las diferencias individuales, como expresión de la riqueza humana y de la tolerancia social.

Para Álvarez Méndez (1999), educar en la diversidad es asumir que alumnos y alumnas de diferentes características deben educarse juntos, debiendo los docentes tomar las decisiones que lo hagan factible desde argumentos de tipo social (educar para compensar desigualdades, ya que no todo el alumnado es capaz de aprender las mismas cosas), de tipo ético (el educador debe promocionar antes que seleccionar), de tipo cultural (la sociedad es diversa y multicultural), de tipo legal (la anterior Reforma decía que la educación debe ser comprensiva y favorecedora de una educación integral), de tipo psicopedagógico (el aprendizaje como proceso de construcción individual en un entorno social) y por último considerar la atención a la diversidad como un proyecto socio-cultural favorecedor del cambio.

Los estudiantes que en estos últimos años acceden a la Universidad son más diversos que los que lo venían haciendo hasta ahora (en relación con los contenidos aprendidos, las actitudes y expectativas sobre la oferta académica, etc.), lo que determina un importante aumento en heterogeneidad y con ello, que los profesores debamos adquirir un mejor conocimiento de ese alumnado.

d) Que el conocimiento del profesor no debe representar el principal saber. Desde la cultura positivista se ha venido incorporando la creencia de un único modelo de verdad, basado en el conocimiento objetivo. Igualmente, desde la tradición de enseñanza academicista, se pone obstáculos al alumno para acceder a la información de una forma directa, se les limita la libertad de pensamiento, acción o movimiento a través de normas impuestas, por considerar que es el profesor el único que dispone de ese conocimiento. Esa cultura reproductora del saber sustituye al conocimiento la experiencial y popular (no considerando los conocimientos previos de los que parte el alumnado, sólo se sigue el conocimiento recogido en los textos como saberes disciplinares fragmentados y que proceden de los propios académicos). Sin embargo, desde un aprendizaje dialógico se considera de que no hay verdades o soluciones definitivas, sino que se deben ir construyendo respuestas provisionales, 
por medio de una dinámica intersubjetiva, en la que el profesor se convierta en un facilitador de ese descubrimiento activo del alumnado.

Este modelo exige que el docente se convierta en facilitador del aprendizaje, organizando las clases a través de grupos que compartan saberes heterogéneos, donde todos aprendemos de todos. Este enfoque comunicativo de enseñanza-aprendizaje contempla: la negociación como una forma de relación respetuosa y digna entre profesor y estudiantes. Desde la libre circulación de ideas, la capacidad individual y colectiva para resolver problemas, la reflexión crítica, el respeto a la dignidad y los derechos de las minorías (Fraile, 1997).

e) La negociación debe representar un procedimiento didáctico que ayude al docente y discente a interactuar e intercambiar mensajes, códigos y discursos. El proceso de negociación: tiene que ver con la capacidad de ponerse de acuerdo entre dos o más partes para realizar una actividad. En este caso, el profesor y estudiantes negociarán el programa de Didáctica de la E.F, dentro de un clima de libertad y confianza, debiendo superar: las relaciones habituales de poder entre profesor-estudiantes, la falta de hábito entre los estudiantes por ofrecer sugerencias al programa, la falta de formación inicial les conduce a posturas prudentes ante el desconocimiento sobre los temas a tratar, la tradicional actitud del alumno que confía en los conocimientos de los profesores y, por ello, no cuestiona dicho programa, etc.

Para hacer posible la negociación, los docentes debemos partir de la creencia de que los estudiantes reúnen una serie de características que posibilitan dicho proceso y que, según Martínez Rodríguez (1995), son:

- los estudiantes son responsables y capaces de generar conocimientos.

- los estudiantes son participantes activos en su propio aprendizaje.

- el profesor debe facilitar que los estudiantes revisen sus propias cuestiones y usen su poder de razonamiento para la comprensión de sus aprendizajes.

- el profesor debe ayudar a los estudiantes a encontrar información, sugiriendo alternativas, proporcionando experiencias o materiales, etc.

- los estudiantes deben encontrar el sentido a las finalidades de su aprendizaje.

- el profesor necesita revisar continuamente su programa, sus resultados, la calidad de la experiencia, etc.

- los estudiantes deben disponer de tiempo para reflexionar sobre lo que hacen, siendo lo que da sentido a dicha actividad y como principal generador del aprendizaje.

La negociación exige que los docentes revisemos y reconozcamos previamente las necesidades e intereses del alumnado, sus conocimientos y experiencias adquiridas, sus creencias, las teorías implícitas, las concepciones, las inquietudes, las actitudes, etc. Gracias a conocer el medio o entorno cultural en el que vive el estudiante, podremos llegar mejor a un acuerdo sobre sus necesidades de aprendizaje.

Si entendemos la negociación como práctica político-social, nuestra actuación académica debe basarse en una cultura formativa que permita que los futuros docentes incluyan en sus programas valores como la equidad, solidaridad, el respeto, la igualdad de oportunidades, etc, como parte fundamental de su actuación. Esto exige que, desde los centros de formación del profesorado, nuestra intervención docente 
se dirija más hacia el desarrollo profesional, como forma integral del futuro educador, que a la transmisión de unos contenidos que, con el paso del tiempo, pierden relevancia y actualidad para su futura actuación docente.

Asimismo, debemos disponer al estudiante hacia una participación activa en el escenario social (saber actuar como futuros ciudadanos). Por ejemplo, es frecuente que los profesores acometamos, como labor profesional, la tarea de diseñar el programa: estableciendo las finalidades, los contenidos a enseñar, la metodología o formas de enseñar y los criterios de evaluación. Rutinas en las que no intervienen los estudiantes, incluso les resultaría extraño que el profesor les pida opinión sobre algunas de ellas.

Como parte final de este proceso, debemos eludir una "falsa negociación" de acuerdos fáciles y rápidos, propios de estructuras organizativas jerarquizadas, en las que se imponen los criterios autoritarios del docente. Tampoco la negociación representa dejar en manos del alumnado todas nuestras responsabilidades académicas y obligaciones docentes. Sin olvidarnos de que la negociación en el ámbito académico es lenta y que la acción docente debe saber actuar ante una realidad educativa compleja y controvertida. Por tanto, es preciso fomentar una acción conjunta y consensuada de todos los agentes educativos que interactuamos en el aula.

\section{UNA EXPERIENCIA DE FORMACIÓN DEMOCRÁTICA CON LOS ESTUDIANTES DE EDUCACIÓN FÍSICA}

Concretamente, en la formación de los futuros docentes de Educación Física, desde la asignatura de Educación Física y su Didáctica, mi propuesta va dirigida a cómo posibilitar desde mis clases un proceso que ayude a que los estudiantes asuman tareas que, hasta ahora y de forma habitual, estaban reservadas para mi como docente. Esta nueva forma de abordar la propuesta curricular, en la formación del profesorado, me permite conocer mejor a los estudiantes: sus necesidades e intereses respecto a la materia, así como compartir con ellos mis principios y compromisos a partir de unos valores como: la libertad, la no discriminación, el tratamiento digno, la solidaridad y el respeto; así como organizar un sistema docente que me ayude a atender la diversidad del alumnado y fomente su autonomía.

a) ¿Cómo se desarrolla la negociación con los estudiantes de Educación Física?

En la primera sesión de clase se solicita a los estudiantes que estén disponibles para intervenir en cuestiones organizativas y pedagógicas de clase, que hasta ahora han venido asumidas únicamente por el docente, como pueden ser la disposición espacial de las aulas, los contenidos a aprender, la metodología o estrategias relativas a cómo aprender, la forma como quieren que se les evalúe, etc. Aspectos que con la negociación democrática pueden variar, desde las necesidades e intereses del alumnado y considerando la complejidad de las diferentes cuestiones que surgen en el aula (Fraile y Martín, 2002).

Si los profesores comenzamos definiendo nuestros principios pedagógicos, ayudará a que los estudiantes expliciten sus compromisos con la materia y con el resto de compañeros. Igualmente, esa negociación facilitará que participen en la definición de los objetivos a lograr, en el diseño de las actividades, en la selección de los contenidos y en los criterios de evaluación. No sería honesto comenzar exigiendo a 
los estudiantes una serie de compromisos, si nosotros como profesores no somos capaces de presentar al grupo cuáles son nuestras intenciones pedagógicas.

En mi caso durante estos años, en los que estoy tratando de modificar mi práctica docente desde una Facultad de Educación, quiero hacer ver a mis estudiantes el compromiso que adquiero con ellos en aspectos para mi esenciales como son: la relación democrática basada en el respeto y el trato digno que se merecen como personas. Tratando de evitar por mi parte que se den situaciones de menosprecio o de ridiculizar en público a aquellos alumnos o alumnas que, por su falta de conocimientos, puedan llevarnos a comportamientos discriminadores.

Es importante que como docentes tratemos de ser coherentes con un tipo de enseñanza activa, donde se reduzcan nuestras intervenciones a favor de una mayor participación del alumnado, basada en la búsqueda y en la resolución de problemas. Donde la transmisión del conocimiento ocupe un lugar secundario, en beneficio de que ellos tengan un contacto más directo y personal con las fuentes bibliográficas, remitiéndoles a artículos o capítulos de libros en los que se recojan cada uno de los temas que se van a desarrollar en clase. Especialmente, debemos animar a aquellos estudiantes con miedo a expresar públicamente sus dudas y que se limitan a reproducir lo dictado por el profesor.

Se trata de impulsar una idea de autogestión en la clase, buscando una mayor implicación de los estudiantes en las decisiones sobre la vida del aula, enseñándoles a organizarse y a asumir responsabilidades relacionadas con el desarrollo del proceso de enseñanza-aprendizaje (funciones que deberán asumir en un futuro como docentes).

Mis propuestas tratan de facilitar el trabajo colaborativo con y entre ellos, considerando que la formación de los grupos se hace a partir de sus propuestas, pero que deberán ir cambiando sus componentes en las diferentes fases del curso, con el fin de que todos aprendan a trabajar con todos. Asimismo, favorecer un clima distendido y de colaboración con los estudiantes, haciendo que se desarrolle y estimule su actitud crítica, no siendo conformista para poder llegar a adoptar un criterio propio ante las cosas.

b) Ayudar a los estudiantes a reconocer: ¿Qué es para ellos ser un buen maestro especialista de Educación Física?

Una de las primeras cuestiones que se recogen al comienzo del curso es ayudar a los estudiantes a definir que es para ello ser un buen maestro de Educación Física. Así como qué tipo de información y formación necesitan para actuar bajo ese ideal de maestro.

En este sentido, es interesante observar cómo entre los contenidos demandados por los estudiantes para su formación son prioritarios aquellos que tienen que ver con los aspectos metodológicos. Por ejemplo: cómo enseñar mejor, cómo comunicarse mejor con sus alumnos y alumnas, cómo aprender a evaluar, etc. Se aprecia en este tipo de respuestas una preocupación por aspectos tradicionalmente académicos o para adquirir competencias técnicas profesionales de utilidad inmediata. Así, demandan la necesidad de aprender unas rutinas (en muchos casos puramente mecánicas), que les ayude a actuar. Sin embargo, estos estudiantes son resistentes a una reflexión sobre la práctica, solicitando respuestas concretas al profesor (como búsqueda de la 
verdad y del conocimiento objetivo). Tampoco valoran sus aportaciones ya sean a nivel individual o en grupo.

Dentro de nuestra labor académica no debemos olvidar que estos alumnos deben:

c) Adquirir unos conocimientos básicos del programa a partir de considerar los descriptores de la didáctica

En este caso, es curioso que los estudiantes cuando se les pregunta sobre qué tipo de conocimientos, como futuros docentes, quieren aprender; en su mayoría, van coincidiendo con los contenidos que ya teníamos previsto atender si hubiésemos optado por un diseño tradicional del programa (es decir, sin considerar las opiniones del estudiante). Aunque también es verdad que algunos de esos contenidos no son expresados con el término preciso y que hay cierta confusión al denominarlos.

Una vez que esos contenidos surgen de los estudiantes, debemos poner en marcha un planteamiento innovador, crítico, cooperativo, etc, que parta de los conocimientos previos, que tengan una incidencia en la práctica, que considere la diversidad del alumnado. Para ello, debemos adaptarnos a un horario fijado por la jefatura de estudios, así como a un calendario que tiene que coordinarse con otras materias y con el Practicum.

No consideramos como necesario diseñar una prueba inicial para conocer los conocimientos previos, sino que de la propia discusión grupal iremos captando información sobre qué les gustaría aprender para ser buenos maestros. Por ejemplo, en pequeños grupos responde a una serie de cuestiones que tienen que ver con delimitar qué es la Educación Física y su futura labor docente: cómo definen E.F., cuáles han sido los motivos para estudiar para maestros de E.F, por qué han dado prioridad a estos estudios sobre la Licenciatura de E.F o sobre otros tipo de estudios, etc. Posteriormente, durante el desarrollo de cada uno de los temas de clase, se diseñan unas preguntas previas para conocer de qué tipo de conocimientos parten los estudiantes, y así ajustar mejor los contenidos a enseñar.

Se pretende estimular la colaboración entre los miembros del grupo, valorando la importancia de su participación en las clases, sometiendoles a situaciones que les ayude a eliminar sus prejuicios respecto a hablar en público y generar sus propias ideas, y sin limitar sus consideraciones críticas. Cada grupo destina un tiempo para contrastar y debatir sobre estas cuestiones planteadas, y un representante de cada uno de los grupos presenta las conclusiones a los demás. Como ejemplo señalamos algunos comentarios de los estudiantes respecto a su participación: "A mi no me resulta fácil compartir ideas con mis compañeros porque pueden saber más que yo, así que me callo y aprendo. En cambio en las clases con los niños, tú eres el que sabes, así que puedes hablar con más tranquilidad".

Este proceso lleva tiempo ya que, debido al desconocimiento de la materia y de los procedimientos a utilizar en su puesta en práctica, inicialmente los estudiantes apenas hacen sugerencias, siendo todo aceptado tal como es presentado por el profesor. Sin embargo, durante el curso y especialmente a la vuelta del Prácticum aumentan sus intervenciones como parte de esa negociación. Asimismo, al disponer de más conocimientos sobre la asignatura y mayor seguridad les anima a ofrecer alternativas al programa. También en las tutorías se dialoga con alguno alumnos 
sobre su escasa participación en los debates, la falta de implicación o iniciativa en el trabajo del aula, se analizan cuestiones educativas relacionadas con: el interés por aprobar en vez de aprender, la preocupación en torno a la evaluación y calificación.

La negociación es parte importante de los procesos de democratización de la enseñanza entre los estudiantes y como parte de su formación, planteándonos la necesidad de que los contenidos, la forma de llevar las clases, los criterios de evaluación, entre otros aspectos, surjan del debate y acuerdo entre profesor y estudiantes. Establecidos los contenidos del curso se repartirán entre los grupos organizados en temas, encargándose ellos de elaborar los materiales que se utilizarán en las clases. La labor del docente será facilitar dicha actuación, ayudándoles a diseñar actividades que tengan sentido para el resto de compañeros. Considerar que al delegar entre los estudiantes esa labor de enseñantes, se consigue que éstos asuman una labor más acorde a su futura labor docente, y con tareas que se ajustan más a los intereses del grupo.

Concretamente, este proceso docente asumido por los estudiantes hace que partan de sus propias ideas, aprendiendo a manifestarlas públicamente desde la revisión de materiales y desde el análisis de sus propias experiencias o vivencias como alumnos. Así en las puestas en común de todo el grupo, los estudiantes deben expresar esos temas con sus propios argumentos, llegando a relacionar sus acciones con planteamientos teóricos. En caso de expresar ideas consideradas como posibles errores, éstas se convierten en puntos de engarce sobre los que se irán construyendo los nuevos conocimientos (Bruner, 1988).

El profesor como facilitador observa y guía la participación de los estudiantes, animándoles a intervenir y colaborando con ellos para que asuman labores de profesor. Con ello, se consigue que los estudiantes den importancia a la información que aportan sus compañeros y se evita que algunos sólo escuchen o escriban lo que dice el profesor, sin considerar las aportaciones del resto. Por ejemplo, solo se consideraba como importante para los estudiantes aquello que el profesor "validaba" escribiéndolo en la pizarra, menospreciando aquello que debatían entre ellos en la clase.

Los materiales que se utilizan en clase (lecturas de artículos), ayudan a que los estudiantes se impliquen en el aprendizaje. De esas lecturas, en principio de forma individual y luego más tarde en grupo, los estudiantes deben extraer la mayor parte de la información sobre el contenido que es objeto de estudio para el debate en las clases. Con ello se busca generar su autonomía, posibilitando que cada uno establezca su propio ritmo de aprendizaje, ya sea desde el trabajo en equipo de clase o en el individual que realizan en casa.

También con una perspectiva crítica, debemos fomentar un tipo de formación del profesorado de Educación Física con interés hacia los temas sociales, donde se analicen las relaciones de poder en la clase, así como los aspectos políticos del proceso enseñanza-aprendizaje. Para ello, esta formación debe situarse en una perspectivas más humanista, que nos acerque a una sociedad más justa, que permita escuchar a los grupos silenciosos y marginados, y aproximar los intereses de la escuela con la sociedad (Sparkes, 1992). Esto representa que:

- El curriculum debe ser percibido de forma holística.

- El docente debe ser el principal agente en la construcción del conocimiento, como portador y creador de cultura. 
- Debemos saber utilizar nuestra experiencia, reconstruyendo y reorganizando el conocimiento.

- Las experiencias previas son importantes en el desarrollo del significado sobre el curriculum.

- La libertad personal es uno de los valores centrales.

- La diversidad y el pluralismo son fines y medios para lograr esos fines.

- Se precisan nuevas formas de lenguaje para construir nuevos significados sobre el curriculum.

- etc.

\section{d) Presentación por parte de los estudiantes de sus compromisos}

Una de las primeras tareas que se pide a los estudiantes es que escriban sus compromisos. Según se recoge en Fraile y Aragón (2003), se deben considerar los tres ámbitos siguientes:

Cognitivos: Compromisos relativos a la adquisición de conocimientos. Cada estudiante se compromete a adquirir el máximo de conocimientos de Educación Física y su Didáctica, que consideran que les ayudará a actuar como mejores docentes en el futuro.

Cada estudiante reconocerá sus conocimientos previos en relación a cada uno de los temas objeto de estudio. Para ayudarles, en ese proceso de reconocimiento, se les presenta una serie de preguntas guía que les facilita esa revisión. Ese conocimiento inicial es posteriormente contrastado con el adquirido durante el curso, así cada uno va tomando nota de sus aprendizajes y de cómo éstos se consiguen.

Recoger información a través de las diferentes fuentes: lectura de artículos o capítulos de libros vinculados a los temas que se consideran, de forma conjunta con los estudiantes, como necesarios para su formación.

Compartir entre compañeros los nuevos conocimientos adquiridos, empleando para ello el diálogo y la discusión colaborativa, ya sea en pequeños grupos o en el gran grupo de clase.

Revisar y valorar el conocimiento adquirido por otros compañeros (heteroevaluación), gracias a la posibilidad de compartir e intercambiar libremente su cuaderno de campo o cuando se les pide emitir un juicio del trabajo de otros compañeros.

Procedimentales: Relativo al proceso que cada estudiante sigue para adquirir sus conocimientos, en la construcción de aprendizajes. Cada estudiante recoge en su cuaderno de campo la información que le servirá para sus informes y trabajos.

Cada uno de los estudiantes viene haciendo uso de diferentes estrategias de recogida de información durante la clase, que incorporan a su cuaderno de campo. Por un lado las ideas seleccionadas de las lecturas, que surgen al compartir y debatir su conocimiento con el resto de compañeros, las presentadas por el profesor, aquellas que se encarga de ampliar cada uno, etc. 
Construcción de un mapa conceptual a partir de las ideas extraídas de las lecturas y de los conocimientos adquiridos. En los mapas se podrán incorporan otros mapas ya elaborados anteriormente.

Realización de informes escritos sobre los temas tratados en las clases: conocimientos previos de cada tema, las ideas adquiridas en lecturas o desde el debate grupal, las aplicaciones didácticas del tema, el mapa conceptual y por último, aquellas preguntas o aspectos que precisan de una posterior revisión después del practicum.

Actitudinales: Disposición que los estudiantes muestran hacia la materia y hacia los compañeros.

Asistencia al mayor numero posible de clases y puntualidad para favorecer el trabajo colaborativo, como una de las claves para la adquisición del conocimiento.

Tener buen nivel de atención y respeto hacia el resto de compañeros.

Participar en los debates y discusiones tanto de pequeño y gran grupo Se valorará más la aportación de nuevas ideas, que la reproducción o aclaración de otras ya presentadas por el profesor u otros compañeros.

Se valorará su capacidad para tomar iniciativas en el grupo, en la elaboración de los informes, en la presentación del trabajo en clase, etc. Así como para ayudar al resto de compañeros cuando algunos de ellos lo precisen.

Después de presentados algunos de los compromisos de los estudiantes, se pasará a desarrollar las actividades propuestas y negociadas con el grupo de estudiantes, que tienen como base el trabajo colaborativo.

e) Ayudar al alumnado en el trabajo colaborativo para favorecer un aprendizaje compartido

Para favorecer ese proceso democratizador, una de las estrategias básicas a desarrollar durante el curso es posibilitar un enseñanza colaborativa que anime a los estudiantes a que se impliquen en la labor docente, a través del intercambio grupal de sus opiniones y discursos (Johnston,1997; Johnson y Otros, 1999).

Inicialmente mostraremos a los estudiantes, a través de ejemplos prácticos, las grandes posibilidades de la metodología colaborativa, a través de poner en común los temas que cada uno conoce, cuestionar los tópicos educativos, favorecer la discusión, contrastar y debatir sobre las divergencias o puntos discrepantes de cada uno, etc. Se trata de que este trabajo grupal evite adoptar posturas neutrales y apostar abiertamente por una visión crítica. También se busca atender la diversidad del grupo, gracias a que todos pueden compartir sus conocimientos e intereses, considerando su actitud-aptitud y disponibilidad, a partir de la utilización de diferentes recursos y materiales (Fraile, 2001).

La distribución y agrupamiento de los estudiantes en el aula se hará según sus necesidades y características, pidiéndoles que varíen periódicamente las estructuras organizativas. Evitar que los grupos siempre estén formados por los mismos, ya que 
es importante que compartan sus conocimientos en grupos más heterogéneos. Durante la clase, se asigna a cada grupo tareas de tal modo que podamos desplazarnos como facilitadores para ayudar a todos ellos. Con la puesta en común del gran grupo, finalizará el trabajo colaborativo, compartiendo y contrastando las conclusiones obtenidas en cada uno de los grupos (Slavin, 1989).

Sobre el uso del espacio y del tiempo, cada propuesta metodológica exige tener presente las características del aula y la duración de las sesiones; por ello, al organizar la clase en grupos debemos disponer de un mobiliario y de un espacio que faciliten esos agrupamientos, así como de sesiones de dos horas de duración que permita un debate con cierto margen de tiempo. Igualmente, este tratamiento didáctico es coincidente con la teoría de la comunicación, ya que posibilita el diálogo y el trabajo de interacción entre los estudiantes. También nos valdremos de un material audiovisual: el vídeo, el retroproyector de transparencias, el cañón de proyección, etc, que complemente o genere temas para la discusión.

Inicialmente se preguntará a los estudiantes sobre el conocimiento o uso de diversas técnicas de estudio como son: el subrayado, la realización de esquemas, cuadros y mapas conceptuales. Comprobar si están habituados a organizar y sintetizar la información de sus lecturas o explicaciones en el aula. Es importante que aprendan a seleccionar el contenido básico del material escrito, relacionándolo con los conocimientos previos de dicho tema. Así como a contrastarlo con otros compañeros, para luego seleccionar lo más relevante.

Debemos provocar entre los estudiantes situaciones de conflicto cognitivo, que les anime a buscar respuestas a las preguntas enunciadas e iniciar el proceso de comunicación, enriqueciendo su trabajo individual, a través de analizar, comparar, añadir, eliminar, sintetizar, etc, hasta llegar a un conocimiento más amplio entre iguales. Deben habituarse a que esa información elaborada de forma individual y grupal, puedan comunicarla al resto de compañeros a través de procesos de interacción y comunicación. En la puesta en común final, todo el grupo de clase compartirá los conocimientos y conclusiones finales. Es el momento cuando el profesor también puede aportar sus experiencias y conocimientos sobre los temas objeto de estudio (ampliando y reformulando lo aportado por el grupo).

Después cada alumno redactará su informe personal, recogiendo los aspectos más significativos tratados en cada una de las fases anteriores y que se han venido escribiendo en los cuadernos de campo (Zabalza, 1991). Además de la información, éstos indicarán las estrategias seguidas, señalando los cambios que se han producido desde los conocimientos previos, hasta el aprendizaje final. Así podrá contrastar los contenidos que poseía al comienzo del proceso, con los obtenidos durante el trabajo colaborativo, como resultado de su aprendizaje. Entre esas actividades destacan las de: revisar los conocimientos previos, leer los artículos y extraer la información más relevante, debatir y compartir información con los compañeros, construir mapas conceptuales, incorporar sugerencias de otros, formular preguntas, buscar aplicaciones didácticas en la práctica, reflexionar sobre el proceso seguido, etc.

El trabajo colaborativo se verá complementado con las reuniones de tutoría, en donde el docente podrá compartir con cada estudiante el conocimiento que tenemos de sus resultados. Para que cada alumno pueda obtener un mejor control de su aprendizaje, se van diseñando unos instrumentos (pruebas con preguntas) con las 
que cada uno podrá revisar su nivel de conocimiento y comprensión de los contenidos. Este tipo de evaluación ayuda a los estudiantes a conocer cómo evolucionan sus aprendizajes, recogiendo en sus posteriores informes de autoevaluación sus valoraciones sobre los resultados obtenidos (siendo importante que ellos valoren lo aprendido o busquen alternativas para aquellos aspectos que no han sabido contestar).

f) Favorecer entre el alumnado procesos de Autoevaluación. La autoevaluación surge como una de las propuestas innovadoras de acuerdo a unos valores democráticos, buscando que el alumno se responsabilice de su desarrollo y resultado. El uso de la autoevaluación exige un alto grado de confianza del profesor hacia el alumno, lo que no exime de establecer unos criterios diseñados y negociados conjuntamente con ellos.

Las razones que justifican la presencia de la autoevaluación son que un alumno debe desarrollar su autonomía y responsabilidad, destacando la labor del docente como facilitador de ese proceso. Con ello también se favorece el desarrollo de valores educativos como son: la autonomía de aprendizaje, la honradez, la dignidad, el análisis crítico y la formación de personas responsables (López Pastor, 1999); (Fraile, 2003).

De cara a que el alumnado pueda autoevaluarse, cada uno de ellos ha podido tomar nota de qué forma evolucionan sus ideas recogidas en sus informes personales: cómo han ido superando las dificultades de aprendizaje, cómo han modificado sus estrategias para aprender, etc. No obstante, este proceso de autoevaluación tiene una relación muy directa con los compromisos que inicialmente cada uno de los estudiantes se propuso conseguir, y la calificación final vendrá al comprobar el cumplimiento de esos compromisos.

\section{g) Pautas para la revisión de los compromisos cumplidos}

A continuación presentaremos algunos criterios para ayudar a cómo cada estudiante puede llegar a valorar sus aprendizajes en cada uno de los aspectos tratados:

Valoración de los aspectos cognitivos:

Cada estudiante valorará la evolución de su aprendizaje desde el conocimiento previo hasta los adquiridos al finalizar cada uno de los temas del curso.

Después de cada informe se realizará una prueba de control de conocimientos. Habrá preguntas que podrán contestar con la ayuda de los cuadernos de campo y otras que tienen un carácter más memorístico. Cada una de las pruebas serán evaluadas y calificadas por los propios alumnos a partir de las respuestas que Ileguen a consensuar como válidas por el conjunto de la clase.

Los estudiantes tendrán un conocimiento de la valoración que hacen de su aprendizaje el profesor y sus compañeros (heteroevaluación), después de que hayan revisado sus informes. En el trabajo grupal se asignará una calificación global para que luego sea repartida, de forma consensuada, según los méritos de cada uno del grupo. También puede haber un reparto por igual entre todos. 
Valoración de los aspectos procedimentales:

Cada estudiante valorará su cuaderno de campo y los informes que realizan otros compañeros (heteroevaluación), considerando de forma negociada los siguientes criterios de valoración:

- Aspectos relativos a una buena presentación.

- Claridad en la redacción y ausencia de faltas ortográficas.

- Si recoge las ideas principales debatidas en clase.

- Si aporta nuevas ideas y argumentos después de leer los informes personales.

- Si cuestiona y completa las ideas debatidas durante la clase.

- Si expresa su visión particular del tema (revisión emocional).

- Si construye nuevos mapas, cuadros o esquemas en donde se sintetiza la información más relevante de cada uno de los temas tratados.

- Si busca diferentes aplicaciones didácticas a las ideas recogidas anteriormente.

- Si genera nuevas preguntas que permita dar continuidad al ciclo de conocimientos.

Valoración de los aspectos actitudinales:

Cada estudiante valorará su nivel de asistencia a lo largo del curso, teniendo como referencia la asistencia obligada al $80 \%$ total de horas de clase.

Valorará su nivel de puntualidad (el referente son los 5’ de cortesía).

Valorará su nivel de atención y respeto hacia el resto de compañeros.

Valorará su participación en los debates y discusiones de pequeño y gran grupo. Distinguir entre la cantidad de participaciones y la calidad de las mismas. La valoración de sus compañeros sobre su participación será un criterio a considerar.

Valorará su disposición a tomar iniciativas dentro del grupo, con objeto de avanzar en los debates. Así como las ayudas que realiza al resto de compañeros.

En diferentes momentos del curso cada estudiante revisará la consecución de sus compromisos, así como la valoración de cada uno ellos, justificando con las actividades realizadas la asignación de una calificación final.

Las actividades que he venido realizando con el objeto de cumplir con todos mis compromisos las he llevado a cabo de una forma sobresaliente: Sobresaliente

Algunas actividades que he venido realizando para cumplir con todos mis compromisos las he llevado a cabo de una forma sobresaliente y el resto de forma notable: Notable

La mayoría de las actividades que he venido realizando con el objeto de cumplir con todos mis compromisos las he llevado a cabo de una forma notable y el resto las considero como aprobadas: Notable 
Algunas de las actividades que he venido realizando con el objeto de cumplir con todos mis compromisos las he llevado a cabo de una forma notable, pero la mayoría las valoro como aprobado: Aprobado

No he podido cumplir con la mayoría de mis compromisos por tanto considero que no merezco el aprobado de la asignatura. Pendiente de negociar con el profesor sus nuevos compromisos para la autoevaluación de Septiembre.

En el caso de que un alumno o alumna considere la posibilidad de obtener Matrícula de Honor deberá presentar en asamblea al resto de compañeros una justificación de dicha petición, los cuales lo considerarán de forma colaborativa y con notación personal si es merecedor o no de dicha calificación.

Esa misma reunión de heteroevaluación cada miembro de clase expondrá su informe final de autoevaluación, la cual ha podido ser previamente contrastada con la opinión del profesor y con los compañeros del pequeño grupo (con los que trabajó durante el curso). Cada uno de los compromisos también pueden ser revisados a partir de tener en cuenta sus progresos, expresando las razones que lo justifiquen. Esas valoraciones solo serán un referente para terminar de definir y concretar su autoevaluación final, ya que primará la calificación que cada uno considere finalmente y de forma totalmente libre.

Por último, destacaré la importancia que esta experiencia está teniendo para favorecer una participación más democrática y responsable entre el alumnado. A pesar de la dificultad que representa llevar a cabo esta forma de evaluar. No obstante, también la evaluación tradicional genera grandes problemas entre el profesorado ya que en muchas ocasiones no se utilizan los criterios o los instrumentos más adecuados para obtener una calificación justa.

Por tanto, adoptamos este criterio de autoevaluación justificado en que representa primar el tratamiento digno y de respeto hacia el alumno, desde una pedagogía crítica en la acción, dejando que cada uno vaya adquiriendo con ello un compromiso responsable con su trabajo y sus resultados. Para concluir, quiero recoger el comentario realizado por un estudiante de E.F. relativo a este tema: "si queremos que nuestros futuros alumnos o alumnas lleguen a ser capaces de autoevaluarse de una manera justa y responsable, debemos conocer dicho proceso desde la práctica para después educar en la justicia y en la igualdad de oportunidades, que les ayude a tomar decisiones basadas en esos principios, mientras tanto resulta complejo seguir esta forma de evaluar".

\section{BIBLIOGRAFÍA}

ÁLVAREZ MÉNDEZ, J.M. (1999) Diversidad y evaluación en educación. En RUBIO y RAYÓN (coord.) Repensar la enseñanza desde la diversidad. Publicaciones MCEP. BOLIVAR, A. (1995) La evaluación de valores y actitudes. Madrid: Anaya. BRUNER, J. ( 1988) Desarrollo cognitivo y educación. Madrid: Morata.

COLL, C. (1990) Aprendizaje escolar y construcción del conocimiento. Barcelona: Paidós. 
CONTRERAS DOMINGO, J. (1995) La construcción de una cultura democrática en la escuela: el papel mediador del docente. Kikiriki, 37, 35-42.

DELORS, J. (1996) La educación encierra un tesoro: Informe a la UNESCO de la Comisión Internacional sobre la Educación para el siglo XXI. Madrid: Santillana.

DEVIS, J. (2001) El currículo oculto y las nuevas orientaciones en el estudio del currículo en la Educación Física. En VÁZQUEZ, B. (coord.) Bases educativas de la actividad física y el deporte. Madrid: Síntesis.

DEWEY, J. (1995) Democracia y educación. Madrid: Morata.

FERNANDEZ BALBOA, J.M. (2000) La Educación Física para el futuro: Tendencias sociales y premisas educativo-profesionales. Tándem, 1. Barcelona: Graó.

FLECHA, R. (1998) Compartiendo palabras. Barcelona: Paidós.

FRAILE ARANDA, A. (1995) El maestro de Educación Física y su desarrollo profesional. Salamanca: Amarú.

FRAILE ARANDA, A. (1997) El trabajo colaborativo como propuesta de formación en Educación Física. En Actas Congreso ICE de Zaragoza.

FRAILE, A.; ARRIBAS, H. y GUTIERREZ, S. (2000) La participación democrática del alumnado en el diseño, puesta en práctica y evaluación del programa de formación: Un proyecto de innovación en la especialidad de Educación Física. En Actas I Congreso Internacional: Docencia Universitaria e Innovación. Barcelona.

FRAILE ARANDA, A. (2001) Revisión y propuestas en la formación de los maestros en didáctica de la Educación Física. En Actas del Congreso de Didácticas específicas. Granada: Universidad de Granada.

FRAILE, A. y MARTíN, R (2002) La Negociación: un paso para democratizar el aula. Actas del XX Congreso Nacional de Educación Física. Guadalajara.

FRAILE, A. y ARAGÓN, A. (2003) La autoevaluación a partir de los compromisos de los estudiantes de E.F. Actas del XXI Congreso Nacional de Educación Física. Tenerife.

HERNÁNDEZ ALVAREZ, J.L. (2000) El futuro de la formación del profesorado de Educación Física. En CONTRERAS JORDAN, O (Coord): La formación inicial y permanente del profesorado de E.F. Ed. Universidad de Castilla La Mancha.

JOHNSON, D.; JOHNSON, R. y HOLUBEC, E. (1999) El aprendizaje cooperativo en el aula. Barcelona: Paidós.

JOHNSTON, M. (1997) El significado de la colaboración más allá de las diferencias culturales. Kikiriki, 46. MCEP.

LOPEZ PASTOR, V. (1999) Prácticas de evaluación en Educación Física. Universidad de Valladolid.

MARCHESI, A. (2000) Controversias en la educación española. Madrid: Alianza.

MARINA, J.A. y VALGOMA, M. (2000) La lucha por la dignidad. Barcelona: Anagrama.

MARTINEZ RODRIGUEZ, J.B. (1995) ¿Tiene el alumnado posibilidad o derecho de realizar innovaciones? En Actas del Congreso Internacional de Didáctica, 96-116. Madrid: Morata.

MARTINEZ RODRIGUEZ, J.B. (1999) Negociación del currículo. Madrid: La Muralla. 
MARTINEZ, J.B. y ARÓSTEGUI, J.L. (2001) La participación democrática del alumnado en los centros de Secundaria. Revista de Educación, 326, 277-295.

MICHAVILA, F. y CALVO, B. (1998) La Universidad española hoy. Propuestas para una política universitaria. Madrid: Síntesis.

PASCUAL, C. (2000) La pedagogía crítica: Una cuestión de ética. En CONTRERAS, O. (coord). La formación inicial y permanente del profesor de Educación Física. Estudios. Universidad Castilla la Mancha.

PEREZ GOMEZ, A. (1992) La formación del profesor como intelectual. En Simposium Internacional Teoría crítica a investigación-acción. Valladolid.

POZO MUNICIO, J.I. (1996) Aprendices y maestros. Madrid: Alianza.

ROGERS, C. (1972) El proceso de convertirse en persona. Barcelona: Paidós.

SLAVIN, R. (1989) Cooperative learning theory. Research and Practice. Englewood Cliffs. N.J.: Prentice-Hall.

SPARKES, A. (1992) Perspectivas curriculares en Educación Física. En CONTRERAS, O y SÁNCHEZ, L.J. (coords). Actas del VIII Congreso Nacional de E.F de Escuelas de Magisterio, 117-155. Universidad de Castilla la Mancha.

VYGOTSKY, L.S. (1979) El desarrollo de los procesos psicológicos superiores. Barcelona: Crítica.

ZABALZA, M.A. (1991) Los diarios de clase. Barcelona: PPU. 